\title{
Guide sommaire de présentation des rapports de surveillance
}

\section{Correspondance : ccdr-rmtc@phac-aspc.gc.ca}

Les rapports de surveillance fournissent des renseignements essentiels sur une maladie ou un problème de santé en fonction de la personne, du moment et du lieu. Ils constituent souvent les fondements servant à déterminer le fardeau de la maladie et peuvent comprendre des renseignements connexes, notamment les tendances en matière de fréquence des facteurs de risque ou les pratiques de prescription. Les rapports de surveillance guident les stratégies destinées à régler des problèmes de santé ciblés, et ils permettent de déterminer la nécessité relative à des soins cliniques ou des mesures de santé publique supplémentaires. II peut s'agir de résumés de rapports plus longs publiés dans la littérature grise, et de plus en plus, ils peuvent être liés à un ensemble de données de surveillance complet. Les rapports de surveillance ont une longueur d'environ 2000 à 2500 mots - excluant le résumé, les tableaux et les références.

En général, le Relevé des maladies transmissibles au Canada (RMTC) appuie l'utilisation des lignes directrices en matière de rapports, y compris celles recueillies par le réseau EQUATOR (Enhancing the QUAlity and Transparency Of health Research) (1). À I'heure actuelle, le réseau EQUATOR ne fournit pas de lignes directrices précises pour les rapports de surveillance, si bien que le RMTC a élaboré ces lignes directrices en fonction d'autres listes de vérification, de lignes directrices pour l'évaluation des systèmes de surveillance (2), ainsi que des dernières tendances en matière de science ouverte.

Le Tableau 1 fournit la liste de vérification du RMTC pour les rapports de surveillance. La Figure 1 illustre un exemple de la façon dont les données de surveillance sont généralement résumées de manière graphique, avec une incidence sur l'axe $y$ et le moment sur l'axe $x$.

Comme pour toutes les soumissions, vérifier les Renseignements à l'intention des auteurs (publiés en janvier chaque année avec le premier numéro de chaque nouveau volume) du Relevé des maladies transmissibles au Canada (RMTC) pour les exigences générales relatives à la préparation et à la soumission des manuscrits.

Tableau 1 : Liste de vérification pour les rapports de surveillance

\begin{tabular}{|c|c|c|}
\hline $\begin{array}{l}\text { Élément de la } \\
\text { déclaration }\end{array}$ & $\mathbf{N}^{01}$ & Description \\
\hline \multicolumn{3}{|l|}{ Titre } \\
\hline Titre & 1 & $\begin{array}{l}\text { Donner un titre qui comprend le nom du problème de santé, la population, le lieu } \\
\text { et le moment. }\end{array}$ \\
\hline \multicolumn{3}{|l|}{ Résumé } \\
\hline Résumé structuré & 2 & $\begin{array}{l}\text { Fournir un résumé structuré, y compris les sous-titres suivants : Contexte, } \\
\text { Objectifs, Méthodologie, Résultats et Conclusion. }\end{array}$ \\
\hline \multicolumn{3}{|l|}{ Introduction } \\
\hline Contexte & 3 & $\begin{array}{l}\text { Résumer la situation actuelle concernant l'état de santé sous surveillance et } \\
\text { déterminer pourquoi il est important. }\end{array}$ \\
\hline Objectifs & 4 & Énoncer l'objectif du rapport de surveillance. \\
\hline \multicolumn{3}{|l|}{ Méthodologie } \\
\hline Milieu & 5 & Décrire le milieu, les lieux et les dates de la période de surveillance. \\
\hline Population & 6 & Décrire la population sous surveillance. \\
\hline Définitions & 7 & $\begin{array}{l}\text { Fournir des définitions pour chaque événement de santé sous surveillance, y } \\
\text { compris les définitions de cas et les interventions de santé publique. }\end{array}$ \\
\hline
\end{tabular}




\begin{tabular}{|c|c|c|}
\hline Sources d'information & 8 & $\begin{array}{l}\text { Décrire toutes les sources de données, y compris l'objectif des systèmes de } \\
\text { surveillance, les données qui ont été recueillies et la façon dont les données ont } \\
\text { été recueillies, transférées et stockées. }\end{array}$ \\
\hline $\begin{array}{l}\text { Données } \\
\text { supplémentaires }\end{array}$ & 9 & $\begin{array}{l}\text { S'il y a lieu, indiquer où les lecteurs peuvent obtenir des renseignements } \\
\text { supplémentaires (p. ex. www.opendata.gc.ca). }\end{array}$ \\
\hline $\begin{array}{l}\text { Qualité des données, } \\
\text { données manquantes et } \\
\text { retards de déclaration. }\end{array}$ & 10 & $\begin{array}{l}\text { Décrire comment la qualité des données a été évaluée. } \\
\text { Expliquer comment les données manquantes ont été traitées. } \\
\text { Si les données sont déclarées selon la date du diagnostic ou de l'apparition des } \\
\text { symptômes, inclure une déclaration quant à savoir si les données pour les } \\
\text { périodes les plus récentes peuvent être révisées. }\end{array}$ \\
\hline Analyse des données & 11 & $\begin{array}{l}\text { Décrire les méthodes d'analyse utilisées en donnant suffisamment de détails } \\
\text { pour permettre à un lecteur bien informé ayant accès aux données originales de } \\
\text { juger de leur pertinence et d'évaluer les résultats déclarés. }\end{array}$ \\
\hline \multicolumn{3}{|l|}{ Résultats } \\
\hline Données descriptives & 12 & $\begin{array}{l}\text { Fournir un résumé des données descriptives, y compris les caractéristiques } \\
\text { démographiques. }\end{array}$ \\
\hline Qualité des données & 13 & $\begin{array}{l}\text { Rapport sur la qualité des données (p. ex. exhaustivité, données manquantes, } \\
\text { sous déclaration). }\end{array}$ \\
\hline Données analytiques & 14 & $\begin{array}{l}\text { Fournir un résumé de l'analyse, y compris (lorsque c'est indiqué) des } \\
\text { estimations des tendances. Les cas échéant, les estimations ponctuelles doivent } \\
\text { comprendre des indicateurs appropriés de mesure des erreurs, par exemple les } \\
\text { intervalles de confiance à } 95 \% \text { (p. ex. changement en pourcentage annuel } \\
\text { moyen utilisé pour décrire les tendances ou les rapports de cotes utilisés pour } \\
\text { décrire les différences de sous-groupes). }\end{array}$ \\
\hline Figures & 15 & $\begin{array}{l}\text { Créer le nombre minimum de figures afin de souligner les principaux résultats. } \\
\text { Créer un titre qui comprend la personne, le lieu et le moment. }\end{array}$ \\
\hline \multicolumn{3}{|l|}{ Discussion } \\
\hline Principaux résultats & 16 & Résumer les principaux résultats en faisant référence aux objectifs de l'étude. \\
\hline Comparaison & 17 & Tenir compte de ces résultats par rapport à la documentation actuelle. \\
\hline Forces et faiblesses & 18 & $\begin{array}{l}\text { Discuter des points forts et des limites de l'étude (qualité des données, } \\
\text { exhaustivité, sources de biais potentielles). }\end{array}$ \\
\hline $\begin{array}{l}\text { Interprétation et } \\
\text { généralisabilité }\end{array}$ & 19 & $\begin{array}{l}\text { Fournir une interprétation globale prudente des résultats en tenant compte des } \\
\text { objectifs, des limites, de la multiplicité des analyses, des résultats issus d'études } \\
\text { semblables, et d'autres données pertinentes. }\end{array}$ \\
\hline Conclusion & 20 & S'assurer que les conclusions abordent l'objectif et font le suivi des résultats. \\
\hline
\end{tabular}


Figure 1 : Taux de cas déclarés d'hépatite $C$ au Canada ${ }^{1}$ selon le sexe, SSMDO ${ }^{2}, 1991-2012$

Taux pour 100000 habitants

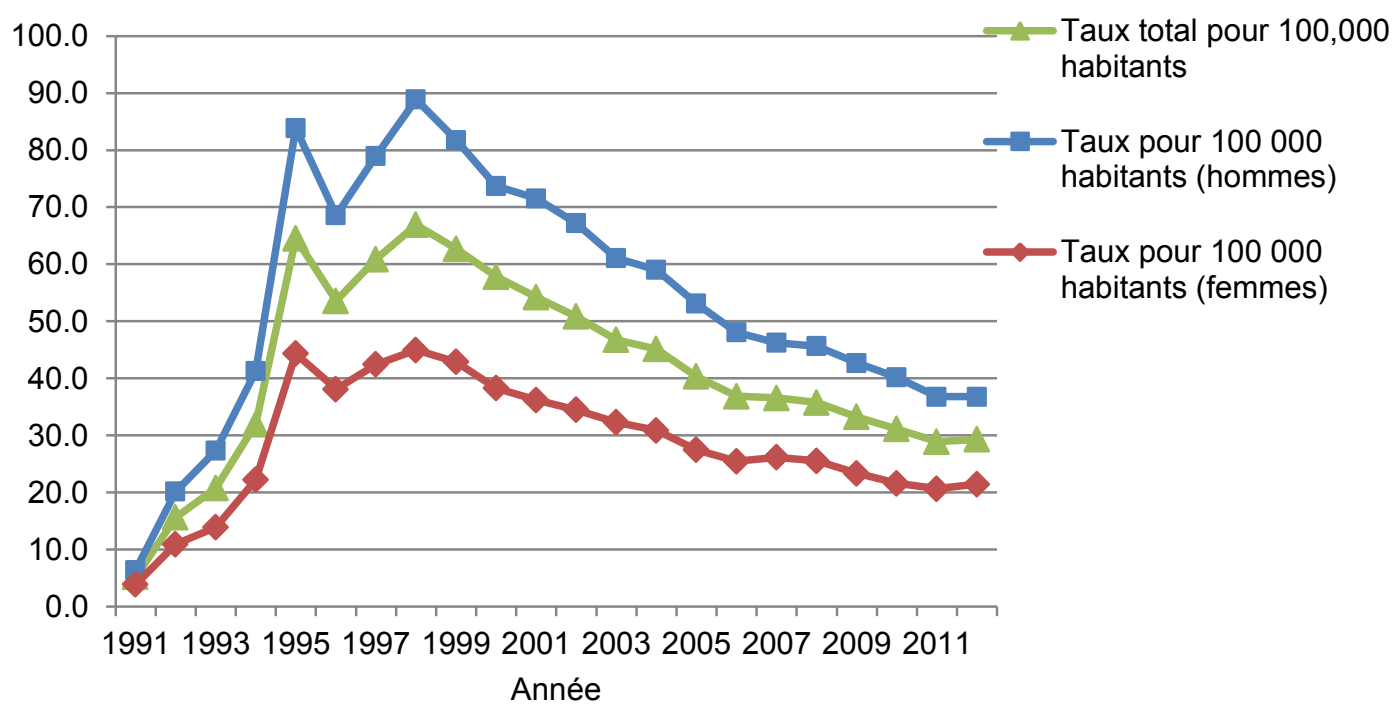

${ }^{1}$ Comprend l'Île-du-Prince-Édouard, l'Ontario, la Saskatchewan, l'Alberta, la Colombie-Britannique, 1991-2012; Terre-Neuve-et-Labrador, le NouveauBrunswick, les Territoires du Nord-Ouest, 1993-2012; le Yukon, 1994-2012; la Nouvelle-Écosse, le Québec, 1996-2012; le Manitoba, 1999-2012; le Nunavut, 1999-2006. Pour le calcul des taux, on a ajusté les dénominateurs des populations pour inclure uniquement les compétences pour lesquelles des données étaient disponibles au cours d'une année donnée.

${ }^{2} \mathrm{SSMDO}=$ Système canadien de surveillance des maladies à déclaration obligatoire

\section{Références}

(1) The Equator Network (Enhancing the QUality and Transparency of health Research)[Internet]. Oxford: EQUATOR Network; 2015. http://www.equator-network.org/.

(2) Klaucke DN, Buehler JW, Thacker SB, Parrish RG, Trowbridge FL, Berkelman RL and the Surveillance Coordination Group. Guidelines for evaluating surveillance systems. MMWR. 1988;37(S-5):1-18. 